\title{
Emphysematous cholecystitis associated with constipation: A case report
}

\author{
Kenji Okumura ${ }^{1,2^{*}}$, Alan T. Lefor ${ }^{2,3}$, Takafumi Maeda ${ }^{1}$, Yoshiharu Takenaka ${ }^{1}$, \\ Toshiyuki Suganuma ${ }^{1}$ \\ ${ }^{1}$ Department of Surgery, Uwamachi General Hospital, Yokosuka, Japan; ${ }^{*}$ Corresponding Author: kenjiokumura@kyudai.jp \\ ${ }^{2}$ Department of Surgery, Tokyo-Bay Urayasu Ichikawa Medical Center, Chiba, Japan \\ ${ }^{3}$ Department of Surgery and the Jichi Medical Simulation Center, Jichi Medical University, Shimotsuke City, Japan
}

Received 21 January 2013; revised 26 February 2013; accepted 1 March 2013

\begin{abstract}
Constipation is one of the most common complaints in the general population, with a wide variety of causes. We present the case of a man with a four-week history of constipation who developed signs and symptoms of acute cholecystitis. Computed tomography scan of the abdomen revealed emphysematous cholecystitis and pericholecystic inflammatory changes extending to the adjacent colon. Emergent cholecystectomy was performed, and bowel function improved after surgery. Chronic inflammation of the gallbladder might be associated with constipation in this patient.
\end{abstract}

Keywords: Constipation; Emphysematous Cholecystitis

\section{INTRODUCTION}

Constipation is one of the most common complaints in the general population, and the causes of chronic constipation are varied. The initial evaluation of chronic constipation should include a careful history and physical examination. The routine use of blood tests, radiography, or endoscopy is limited to selected individuals, such as patients with hematochezia, weight loss of $\geq 10$ pounds, a family history of colon cancer or inflammatory bowel disease, anemia, positive fecal occult blood tests, or acute onset of constipation in elderly persons [1,2]. We present the case of an elderly man with a four week history of constipation who developed signs and symptoms of acute cholecystitis.

\section{CASE PRESENTATION}

A 76-year-old man with a history of hypertension presented with a four-week history of constipation and a 2- day history of worsening abdominal pain with nausea. He had no history of diabetes mellitus or weight loss over the previous 6 months. On examination, he had a temperature of $37.8^{\circ} \mathrm{C}$, blood pressure of $136 / 82 \mathrm{mmHg}$, and a heart rate of $84 \mathrm{bpm}$. He appeared neither icteric nor anemic. Abdominal examination showed mild tenderness in the right upper quadrant and a positive Murphy's sign. Laboratory results included: white cell count of 12,300/ul (normal: $3100-8800 / \mathrm{ul}$ ), C-reactive protein $7.0 \mathrm{mg} / \mathrm{dl}(0-0.25 \mathrm{mg} / \mathrm{dl})$, platelet count of $100 \times 10^{9} / \mathrm{ul}$ $\left(140-440 \times 10^{9} / \mathrm{ul}\right)$, aspartate transaminase $21 \mathrm{IU} / 1$ (13 $33 \mathrm{IU} / 1)$, alanine transaminase $15 \mathrm{IU} / \mathrm{L}$ (6 - $27 \mathrm{IU} / \mathrm{l})$, and alkaline phosphatase $198 \mathrm{IU} / \mathrm{L}$ (115 - $359 \mathrm{IU} / \mathrm{l})$. In addition, total bilirubin was $2.71 \mathrm{mg} / \mathrm{dL}(0.3-1.5 \mathrm{mg} / \mathrm{dL})$, gamma glutamyl transferase $13 \mathrm{IU} / \mathrm{L}(10$ - $60 \mathrm{IU} / \mathrm{L})$. Abdominal ultrasonography revealed an enlarged gall bladder containing stones. Computed tomography scan of the abdomen showed an enlarged gall bladder, multiple calcified gallstones, pericholecystic fat stranding, an air-fluid level within the gallbladder lumen, and ascending colon wall thickening with incomplete obstruction (Figures 1(a) and (b)). The diagnosis of emphysematous cholecystitis was made, and emergent cholecystectomy performed. The gallbladder was gangrenous and emphysematous. The patient was treated with cefoperazone-sulbactam (1 g every $12 \mathrm{~h}$ ) and had an uneventful recovery. Gallbladder tissue culture showed Klebsiella pneumoniae and Clostridium perfringens, a known gas-forming organism. Constipation improved after the cholecystectomy and colonic wall thickening also improved based on $\mathrm{CT}$ scan evaluation at one month.

\section{DISCUSSION}

Cholecystitis is one of the common causes of abdominal pain, but acute emphysematous cholecystitis is a relatively rare condition, which predominantly affects elderly men [3]. The mortality rate for emphysematous cholecystitis is relatively high due to the increased risk of 


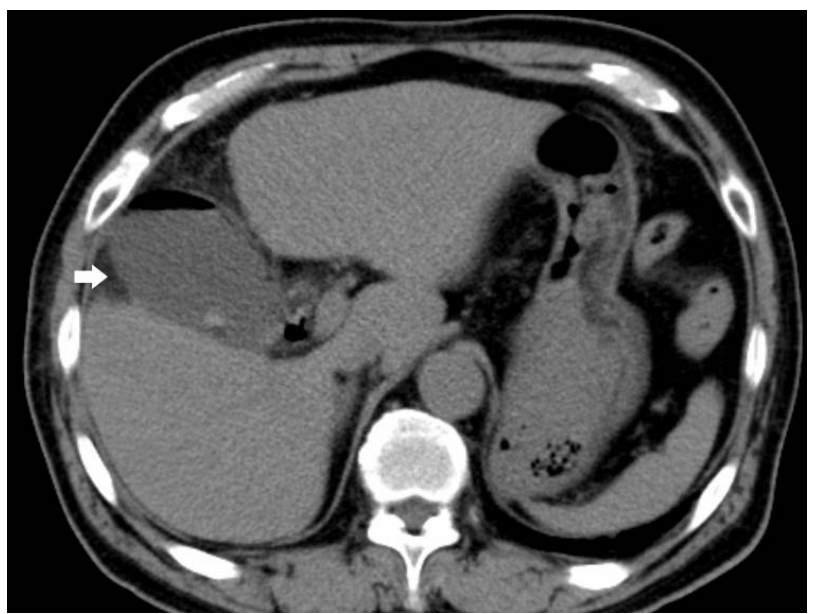

(a)

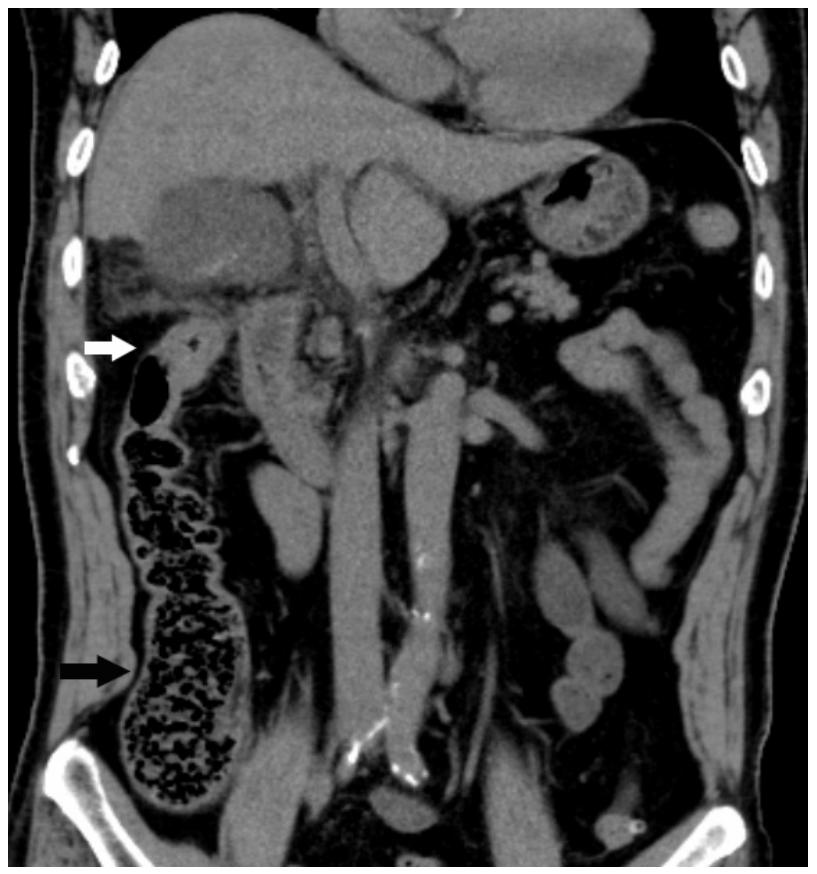

(b)

Figure 1. (a) Computed tomographic scan shows intraluminal gas and mural thickening of the gallbladder (white arrow); (b) Coronal reformatted computed tomography scan shows mural thickening of the gallbladder, pericholecystic inflammatory changes extending to the adjacent colon (white arrow) with stool in the proximal colon (black arrow).

gallbladder wall gangrene and perforation [3]. This patient with emphysematous cholecystitis had an associated history of four weeks of constipation. Computed tomography scan of his abdomen revealed emphysematous cholecystitis, pericholecystic inflammatory changes extending to the adjacent colon and stool in the proximal colon. This might suggest that inflammation of the gallbladder interfered with bowel function and resulted in constipation. After treatment of cholecystitis, bowel function improved and the complaint of constipation resolved. The wall thickening of the adjacent colon and inflammatory changes were improved on CT-scan at one month. Chronic inflammation of the gallbladder might be associated with this patient's constipation.

Chronic colonization by organisms is well known, and the impact of colonization was considered. Chronic cholecystitis was confirmed based on the history and pathological specimen. However, there is no literature describing a case of chronic cholecystitis affected by constipation. The time from onset is the key factor in the treatment of emphysematous cholecystitis, and early diagnosis and emergent operation are essential for good outcomes [3-5]. Fortunately, this patient was successfully treated, but emphysematous cholecystitis is a lethal disease with a high mortality rate. To diagnose early, one must keep biliary tract disease in mind as one of the differential diagnoses when we see elderly patients with constipation.

\section{CONCLUSION}

We present the case of emphysematous cholecystitis with a four week history of constipation. When we see elderly patients with constipation, it is important to keep biliary tract disease in mind as one of the associated causes.

\section{REFERENCES}

[1] Saures, N.C. and Ford, A.C. (2011) Prevalence of, and risk factors for, chronic idiopathic constipation in the community: Systematic review and meta-analysis. American Journal of Gastroenterology, 106, 1582-1591. doi:10.1038/ajg.2011.164

[2] Rao, S.S., Ozturk, R. and Laine, L. (2005) Clinical utility of diagnostic tests for constipation in adults: A systematic review. American Journal of Gastroenterology, 100, 16051615. doi:10.1111/j.1572-0241.2005.41845.X

[3] Moanna, A., Bajaja, R. and del Rio, C. (2006) Emphysematous cholecystitis due to Salmonella derby. The Lancet Infectious Diseases, 6, 118-120. doi:10.1016/S1473-3099(06)70385-1

[4] Garcia-Sancho Tellez, L., Rodriguez-Montes, J.A., Fernandez de Lis, S. and Garcia-Sancho Martin, L. (1999) Acute emphysematous cholecystitis. Report of twenty cases. Hepatogastroenterology, 46, 2144-2148.

[5] Hazey, J.W., Brody, F.J., Rosenblatt, S.M., Brodsky, J., Malm, J. and Pronsky, J.L. (2001) Laparoscopic management and clinical outcome of emphysematous cholecystitis. Surgical Endoscopy, 15, 1217-1220. doi: $10.1007 / \mathrm{s} 004640080018$ 